\title{
Blue hypertext is a good design decision: No perceptual disadvantage in reading and successful highlighting of relevant information.
}

\author{
Benjamin Gagl Corresp. 1,2 \\ 1 Department of Psychology, Johann Wolfgang Goethe Universität Frankfurt am Main, Frankfurt am Main, Germany \\ 2 Center for Individual Development and Adaptive Education of Children at Risk (IDeA), Frankfurt am Main, Germany \\ Corresponding Author: Benjamin Gagl \\ Email address: gagl@psych.uni-frankfurt.de
}

Background. Highlighted text in the Internet (i.e. Hypertext) is predominantly blue and underlined. The perceptibility of these hypertext characteristics was heavily questioned by applied research and empirical tests resulted in inconclusive results. The ability to recognize blue text in foveal and parafoveal vision was identified as potentially constrained by the low number of foveally centered blue light sensitive retinal cells. The present study investigates if foveal and parafoveal perceptibility of blue hypertext is reduced in comparison to normal black text during reading.

Methods. A silent-sentence reading study with simultaneous eye movement recordings and the invisible boundary paradigm, which allows the investigation of foveal and parafoveal perceptibility, separately, was realized (comparing fixation times after degraded vs. un-degraded parafoveal previews). Target words in sentences were presented in either black or blue and either underlined or normal.

Results. No effect of color and underlining, but a preview benefit could be detected for first pass reading measures. Fixation time measures that included re-reading, e.g., total viewing times, showed, in addition to a preview effect, a reduced fixation time for not highlighted (black not underlined) in contrast to highlighted target words (either blue or underlined or both).

Discussion. The present pattern reflects no detectable perceptual disadvantage of hyperlink stimuli but increased attraction of attention resources, after first pass reading, through highlighting. Blue or underlined text allows readers to easily perceive hypertext and at the same time readers re-visited highlighted words longer. On the basis of the present evidence blue hypertext can be safely recommended to web designers for future use. 
1 Blue hypertext is a good design decision: No perceptual disadvantage in reading and successful

2 highlighting of relevant information.

3

4

Benjamin Gagl

5 Department of Psychology, Goethe University Frankfurt \& Center for Individual Development and Adaptive Education of Children at Risk (IDeA), Frankfurt am Main

7

8

9 Address for correspondence:

10 Benjamin Gagl

11 Department of Psychology

12 Goethe University Frankfurt

13 Theodor-W.-Adorno-Platz 6

1460323 Frankfurt/Main, Germany

15 Tel.: 00496979835336

16 E-mail: gag1@psych.uni-frankfurt.de

17 Word count: 2999 (excluding Abstract and References) 
20 Background. Highlighted text in the Internet (i.e. Hypertext) is predominantly blue and

21 underlined. The perceptibility of these hypertext characteristics was heavily questioned by

22 applied research and empirical tests resulted in inconclusive results. The ability to recognize blue 23 text in foveal and parafoveal vision was identified as potentially constrained by the low number 24 of foveally centered blue light sensitive retinal cells. The present study investigates if foveal and parafoveal perceptibility of blue hypertext is reduced in comparison to normal black text during 26 reading.

27 Methods. A silent-sentence reading study with simultaneous eye movement recordings and the invisible boundary paradigm, which allows the investigation of foveal and parafoveal perceptibility, separately, was realized (comparing fixation times after degraded vs. un-degraded parafoveal previews). Target words in sentences were presented in either black or blue and either underlined or normal.

32 Results. No effect of color and underlining, but a preview benefit could be detected for first pass reading measures. Fixation time measures that included re-reading, e.g., total viewing times, showed, in addition to a preview effect, a reduced fixation time for not highlighted (black not underlined) in contrast to highlighted target words (either blue or underlined or both).

Discussion. The present pattern reflects no detectable perceptual disadvantage of hyperlink stimuli but increased attraction of attention resources, after first pass reading, through highlighting. Blue or underlined text allows readers to easily perceive hypertext and at the same time readers re-visited highlighted words longer. On the basis of the present evidence blue hypertext can be safely recommended to web designers for future use. 
43

44

45

46

47 The Internet plays an important role in our daily life. One of the first but also most critical 48

\section{Introduction} advantages of the Internet is the use of hypertext. Hypertext allows the web designer to efficiently link important snips of text to additional information. Thus, hypertext works by replacing referencing in printed text and eliminating effortful literature searches. The most common implementation of hypertext, embedded as blue underlined text, was prominently criticized (Nielson, 1999). It was argued that choosing blue as text color is a poor choice as only about $2 \%$ of retinal cells are sensitive to light with short wave-lengths, which give rise to the perception of blue. As a consequence, blue hypertext might reduce reading speed due to hampered foveal processing. This would be unfortunate since it would limit the general increase of effectiveness of text processing introduced by hypertext. In addition, color sensitive retinal cells are most prominent in the fovea of the eye and their number decreases massively towards para- and extra-foveal regions of the retina. This massive reduction of color sensitive cells towards the para- and extra-foveal regions might also decrease the possibility to extract relevant parafoveal information from colored text in reading. In general, parafoveal preprocessing typically increases reading speed (for a review see Schotter, Angele, \& Rayner, 2012). Therefore a decrease in reading speed resulting from both reduced parafoveal preprocessing and foveal perception would be drastic when considering how often hypertext is perceived. Such a decrease in reading efficiency would indicate that the use of blue underlined hypertext could not be recommended.

Recently, Fitzsimmons, Weal, and Drieghe (2013) investigated the influence of colored words on eye movement measures and found a reduced skipping probability (i.e., the probability 
68 of not fixating a word) of blue words, which were embedded in single line sentences. Their

69 paradigm allows access of the combined foveal and parafoveal processing during silent reading

70 of sentences. They found a reduced reading speed, in contrast to black text, for words written in

71 gray but not for words written in other colors (e.g., blue). This finding indicates that contrast

72 (black vs. gray) but not color (e.g., black vs. blue) hampers reading speed. For words presented

73 in saturated colors (e.g., blue) they found a reduced skipping probability in contrast to black-

74 presented words. In their second experiment, Fitzsimmons et al., (2013) did not find reduced

75 skipping for blue colored words. In this experiment, the blue colored words were embedded in a

76 paragraph, which was presented in a realistic online context (i.e., Wikipedia page). No effect of

77 color on skipping was found but an interaction of a word frequency manipulation (seldom vs.

78 common words) and the text color could be detected in late eye-movement measures of go-past-

79 time (i.e., the sum of all fixations after the target word was fixated first and before the next word

80 in the sentence is fixated; this includes fixations on words previous to the target word) and total

81 viewing time (i.e., all fixation durations on the target word).

82 The finding in the first experiment from Fitzsimmons et al. (2013) of a reduced skipping

83 rate for colored words can be interpreted in two ways: Either bottom-up perceptual processes are

84 hampered due to a reduced parafoveal perceptibility of blue words, increasing the fixation

85 probability. The reduced visual information extraction would indicate that the lack of parafoveal

86 blue light sensitive retinal cells results in less reliable visual information extraction. This then

87 would reduce, first, information extraction from parafoveal words and, second, reading speed as

88 a consequence of hampered word recognition processes. The second way to interpret the finding

89 of a decreased skipping probability for blue words is that top-down processes, which increase the

90 fixation probability, reflect the learned association of hypertext to informative content. Therefore, 
91 highlighted words might attract additional attentional resources in contrast to un-highlighted

92 words. The finding of the second experiment from Fitzsimmons et al. (2013) is in line with this

93 highlighting hypothesis. There they found no skipping effect difference between blue and black

94 written text but showed that late eye movement measures were prolonged in case seldom, blue

95 written words were fixated. Furthermore, this finding might indicate that such a top-down

96 process would need time to evolve. This is consistent with previous findings showing that late

97 eye-movement measures (e.g. total viewing time) are increased in case top-down processes like

98 context demands or effortful reading instructions were manipulated (Radach, Huestegge \& Reilly, 99 2008).

To differentiate between these hypotheses the present study realized an invisible

101 boundary paradigm (Rayner, 1975; for a revised version of the paradigm see Gagl, et al., 2014).

102 This paradigm allows researchers to estimate the parafoveal preview benefit by contrasting

103 fixation times after perfect previews (no manipulation) in contrast to degraded previews (limiting

104 preview benefits). The task of the participant is reading sentences silently as if they were reading

105 a book or newspaper (i.e. as natural as possible). An invisible boundary is placed before a target

106 word (see Figure 1a). When the invisible boundary is crossed by a saccade the change from a

107 degraded to an un-degraded target word presentation is realized during the eye movement. The

108 increase of reading speed after the parafoveal presentation of a normal word compared to the

109 condition with a degraded word is interpreted as the parafoveal preview benefit. In our Gagl et al.

110 (2014) study we were the first to use degraded parafoveal previews and showed such a degraded

111 word before fixation elicited only less preprocessing benefit but no preprocessing costs. This is

112 crucial as for parafoveal masks (e.g. different letter or X-masks), which were used originally

113 instead of degradation, preview costs were described (see Hutzler, et al., 2013; Kliegl, 
114 Hohenstein, Yan \& McDonald, 2013). These costs potentially magnify parafoveal-preprocessing

115 effects due to undesired processing of the mask. The boundary paradigm cannot be optimally

116 implemented in case the skipping rate is expected to vary largely between conditions, as the

117 estimation of the preview benefit relies on the fixation times on the target word. To realize high

118 target word fixation rates, the predictability out of the sentence context was held low for the

119 target words, which decreases skipping probabilities (Fitzsimmons, \& Drieghe, 2013; Hawelka,

120 Schuster, Gagl, \& Hutzler, 2015). Therefore, low skipping rates, at the best-case floor effects, are

121 expected to reduce the probability of finding differential effects in this measure. The effects of

122 the present manipulations are expected in the fixation time measures of first fixation duration (i.e.

123 the duration of the initial fixation), gaze duration (i.e., the summated fixation duration of all

124 fixations during the first encounter), go-past time and total viewing time. In addition, two

125 manipulations were implemented that allow to differentiate between the perceptual bottom-up

126 (i.e. reduced reading speed for blue text) and the highlighting top-down hypothesis (i.e. more

127 top-down attention is allocated to highlighted text). This was realized by manipulating color

128 (blue vs. black) and underlining (underlined vs. not underlined) of the target words. The resulting

129 design included the factors color, underlining and parafoveal degradation (see Fig. 1).

130 In case parafoveal bottom-up processing of blue stimuli is limited, a reduced parafoveal

131 preview benefit in contrast to black words is expected. Limited foveal bottom-up processing of

132 blue text would result in higher fixation times of blue vs. black target words. This should be the

133 case irrespective of underlining or parafoveal preview. The underlining aspect is crucial here as

134 longer fixation durations for blue text in contrast to underlined black target words would exclude

135 the possibility that such a difference would be the result of highlighting. Both parafoveal and

136 foveal findings would indicate a hampered bottom-up processing of blue hypertext. Alternatively, 
137 if top-down processes that originate from highlighting influence the reading behavior, than the

138 un-highlighted condition (i.e., black not-underlined targets) should receive less attention. In

139 contrast, the highlighted words, blue not-underlined, blue underlined and black underlined

140 targets, should receive additional attention reflected in longer fixation duration measures. If color

141 or underlining results in independent highlighting effects (e.g. underlining effect when the target

142 word is not colored), one can even recommend text underlining or coloring independently for

143 hypertext use.

144

145

Methods

146

147 Participants

148 Forty native German-speaking students (24 female; mean age: 23:2 years:month; standard

149 deviation: 2:0) with normal reading speed measured by the unpublished adult version of the

150 Salzburger-Lese-Screening (SLS; Auer, Gruber, Mayringer \& Wimmer, 2004; for the current

151 state of the adult version see Gagl, Hawelka, \& Hutzler, 2014) and normal or corrected-to-

152 normal vision participated. One additional participant was excluded due to very slow reading

153 (Percentile $<16$ ). Participants gave informed consent and the research was approved by the

154 ethics board of the University of Salzburg (EK-GZ: 20/2014).

155

156 Apparatus

157 Movements of the right eye were recorded with a sampling rate of 2,000 Hz (EyeLink CL eye-

158 tracker, SR-Research, Canada). Participants were seated about $52 \mathrm{~cm}$ in front of a CRT monitor

159 (150-Hz refresh rate; screen resolution of $1024 \times 768$ pixels) and a forehead and chin rest 
160 stabilized their heads. The display change latency of the experimental setup was below $15 \mathrm{~ms}$

161 (for details see Richlan et al., 2013).

162

163 Material

164 The manipulation of color and highlighting was realized with five letter target words embedded

165 in sentences, which were matched on the most important word characteristics (e.g., orthographic

166 similarity: OLD20, Yarkoni et al., 2008; word frequency: SUBTLEX database, Brysbaerd et al.,

167 2011; and predictability from sentence context, e.g., Kliegl, Grabner, Rolfs \& Engbert, 2004).

168 Furthermore eight different versions of all sentences $(\mathrm{N}=320)$ allowed the presentation of each

169 sentence in one of the eight conditions (40 per condition; Fig. 1a). An equal number of

170 participants $(n=5)$ were assigned to each of the eight versions of the sentences (Latin square

171 design). The parafoveal preview manipulation was realized by randomly replacing $45 \%$ of the

172 black or blue pixels of the presented letters (for details see Gagl, et al., 2014). This procedure

173 distorted the parafoveal percept of the target words without inhibiting lexical processing. The

174 sentences were presented in a mono-spaced font (single character width: $0.3^{\circ}$ of visual angle)

175 and target words were never at the first, second, or final position of the sentences (All stimuli are 176 available under: https://osf.io/8c57w/).

177

178 Procedure

179 A 3-point calibration of the eye tracker preceded the experiment. Fixating between two vertical

180 lines in the left margin of the monitor triggered sentence presentation in such a way that the

181 participants' fixation was at the center of the sentence's first word. The students read silently for 182 comprehension. Fixating an ' $\mathrm{x}$ ' in the lower right corner of the screen terminated the trial and 
183 removed the sentence from the screen. After the presentation of about 50 randomly selected

184 sentences, a question mark appeared on the screen and the experimenter orally presented

185 comprehension questions, which the participants almost always answered correctly $(\mathrm{M}=98 \%)$.

186 All words after the target word were visually degraded to minimize potential influences

187 of these words (i.e., particularly of $n+2$, with $n+1$ being the target word; see Kliegl, Risse \&

188 Laubrock, 2007 and Fig. 1a). After crossing the invisible boundary at the end of the pre-target

189 word, the target word and the remainder of the sentence were presented un-degraded (see Fig.

190 1a). Ten practice trials preceded the experiment. Recalibration was conducted after the practice

191 trials, after a break halfway through the experiment, and when the fixation control at the start of

192 a trial failed.

193

194 Data Treatment and Analyses

195 Skipping probabilities, first fixation durations, gaze durations, and total viewing time are

196 reported. First fixation durations, gaze durations and total viewing times shorter than $80 \mathrm{~ms}$ were

197 removed from the data (for each measure $<1 \%$ of the data). Data analysis was administered with

198 linear mixed effect models (LMMs) for the log-transformed fixation timing measures and

199 generalized linear mixed effect models (GLMMs) for the skipping probability (this analysis is

200 best suited to estimate binary data: skipped vs. fixated) with the lme4-package (Bates, et al.,

201 2015) in R. G/LMMs are suited for analyzing unbalanced data (e.g., due to skipping of target

202 words). Color, underlining, degradation and all interactions were included in the models as fixed

203 effects (treatment contrast in relation to the baseline of black, not underlined, degraded words).

204 Random effects were estimated for the intercepts of both participants and items. In addition, the

205 random slopes for the fixed factors were added to the model until an additional parameter did not 
206 allow the model to converge. In case adding another level to the random effect structure resulted 207 in a not converging model, one of the other two factors was introduced into the model and the 208 model was refitted. If two models with the same number of random slope estimates converged, 209 an ANOVA was used to compare the model fits and allowed to decide which model estimated 210 the data better. This procedure resulted in the additional estimation of the random slope of 211 degradation on the random effect of participant for the skipping probability. For the first fixation 212 duration, the random slopes of degradation and color were estimated for the random effect of 213 participant. For the gaze duration, the random slopes of underlining, color and degradation on the 214 random effect of participant and the random slope of degradation on the random effect of item 215 were estimated. For the go-past times, the random slopes of underlining, color and degradation 216 on the random effect of participant and the random slope of underlining on the random effect of 217 item were estimated. For the total viewing time the random slopes of underlining, color and 218 degradation on the random effect of participant were estimated. With this procedure, the most 219 conservative converging models were selected (Data and analysis scripts are available under: 220 https://osf.io/8c57w/). 
a

Das gelbe Blatt wird vom Wind davon getragen.

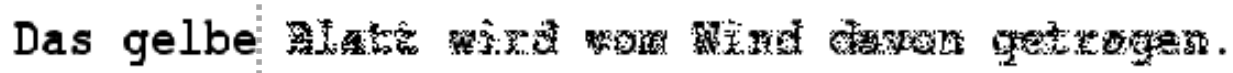

Das gelbe Blatt wird vom Wind davon getragen.

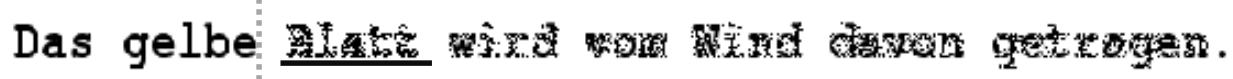

Das gelbe Blatt wird vom Wind davon getragen.

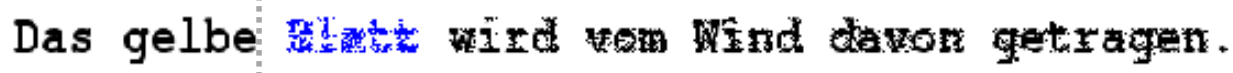

Das gelbe Blatt wird vom Wind davon getragen.

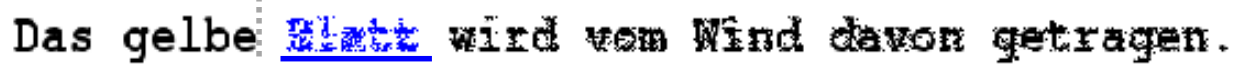

b
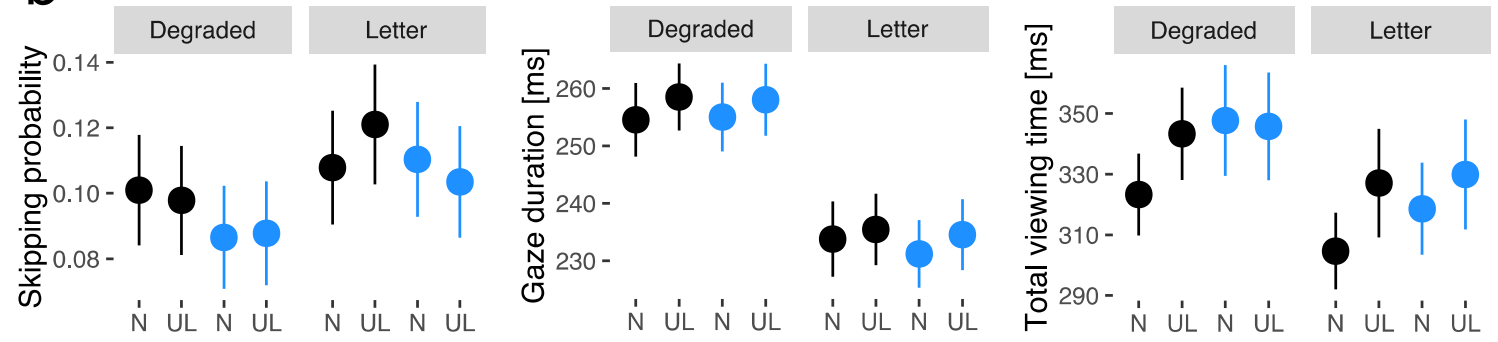

222
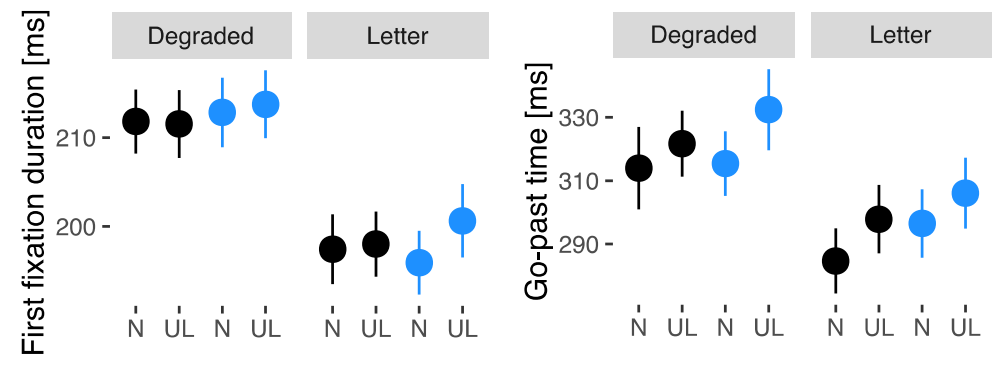

223 Figure 1. Stimulus presentation and eye movement data. (a) Example sentence for all eight

conditions (black not-underlined, black underlined, blue not-underlined, blue underlined in

225 degraded and un-degraded versions) with the embedded target word Blatt (English: leaf). Before

226 each target the gray line indicated the invisible boundary, which triggered the display change

227 from degraded to un-degraded presentations in case a saccade crossed the boundary. The effect

228 of parafoveal degradation can be visualized by introspection. Compare the percept of the target

229 word after fixating either on the pre-target word of a degraded or an un-degraded target word. (b) 
230 Means and standard errors (vertical bars) of skipping probability, first fixation duration, gaze

231 duration, go-past time, and total viewing time. Blue dots indicated blue words and black dots

232 black words. $U L$ indicates underlined presentation and $N$ indicates normal presentation.

233 
Table 1. Intercepts and fixed effects of LMM analyses for skipping probability, first fixation duration, gaze duration, go-past times and total viewing time (all timing measures were log transformed).

\begin{tabular}{|c|c|c|c|}
\hline \multirow[b]{2}{*}{ Skipping probability } & Fixed effects & $S E$ & \multirow[b]{2}{*}{ Z-values } \\
\hline & & & \\
\hline Intercept & -2.61 & 0.19 & 13.63 \\
\hline Degradation (Deg) & 0.14 & 0.13 & 1.08 \\
\hline Color (Col) & -0.18 & 0.13 & 1.48 \\
\hline Underlined (Undl) & -0.04 & 0.12 & 0.32 \\
\hline Deg X Col & 0.22 & 0.17 & 1.25 \\
\hline Deg X Undl & 0.18 & 0.17 & 1.09 \\
\hline Col X Undl & 0.05 & 0.18 & 0.30 \\
\hline Deg X Col X Undl & -0.28 & 0.24 & 1.14 \\
\hline First fixation duration & & & $t$-values \\
\hline Intercept & 5.31 & 0.017 & 314.53 \\
\hline Deg & -0.072 & 0.012 & 6.12 \\
\hline $\mathrm{Col}$ & 0.004 & 0.011 & 0.33 \\
\hline Undl & -0.001 & 0.010 & 0.10 \\
\hline Deg X Col & -0.009 & 0.014 & 0.65 \\
\hline Deg X Undl & 0.004 & 0.014 & 0.24 \\
\hline Col X Undl & 0.007 & 0.014 & 0.50 \\
\hline Deg X Col X Undl & 0.011 & 0.020 & 0.54 \\
\hline \multicolumn{4}{|l|}{ Gaze duration } \\
\hline Intercept & 5.47 & 0.03 & 190.87 \\
\hline Deg & -0.098 & 0.015 & 6.73 \\
\hline $\mathrm{Col}$ & 0.007 & 0.012 & 0.59 \\
\hline Undl & 0.021 & 0.013 & 1.59 \\
\hline Deg X Col & -0.013 & 0.017 & 0.79 \\
\hline Deg X Undl & -0.015 & 0.017 & 0.91 \\
\hline Col X Undl & -0.012 & 0.017 & 0.69 \\
\hline Deg X Col X Undl & 0.021 & 0.024 & 0.89 \\
\hline \multicolumn{4}{|l|}{ Go-past time } \\
\hline Intercept & 5.62 & 0.04 & 151.07 \\
\hline Deg & -0.103 & 0.015 & 6.32 \\
\hline Col & 0.026 & 0.016 & 1.69 \\
\hline Undl & 0.037 & 0.015 & 2.26 \\
\hline Deg X Col & 0.008 & 0.016 & 0.40 \\
\hline Deg X Undl & -0.001 & 0.020 & 0.05 \\
\hline Col X Undl & -0.004 & 0.020 & 0.20 \\
\hline Deg X Col X Undl & 0.000 & 0.029 & 0.01 \\
\hline \multicolumn{4}{|l|}{ Total viewing time } \\
\hline Intercept & 5.63 & 0.04 & 127.03 \\
\hline Deg & -0.080 & 0.015 & 5.32 \\
\hline $\mathrm{Col}$ & 0.031 & 0.017 & 1.77 \\
\hline Undl & 0.046 & 0.018 & 2.59 \\
\hline
\end{tabular}




\begin{tabular}{lccc} 
Deg X Col & -0.011 & 0.021 & 0.53 \\
Deg X Undl & -0.028 & 0.021 & 1.34 \\
Col X Undl & $\mathbf{- 0 . 0 4 4}$ & $\mathbf{0 . 0 2 1}$ & $\mathbf{2 . 0 9}$ \\
Deg X Col X Undl & 0.040 & 0.030 & 1.33 \\
\hline
\end{tabular}

Note. Significant fixed effects are highlighted in bold numerals

234 As expected skipping probability, presented in Figure 1b, was not reliably affected by color,

235 underlining or degradation (see Table 1). The present study reports low skipping probabilities

236 between 8 and $12 \%$, when compared to the Fitzsimmons study with skipping probabilities up to

$23727 \%$. Contrasting the two studies differences in the word frequency of the target words might

238 explain the overall difference in skipping probability as in the present study words with low

239 frequency were used and Fitzsimmons used words with higher frequencies. The result of the

240 present study indicates that fixation rates of the target words are comparable indicating a floor

241 effect for cognitive influences on word skipping.

242 In contrast, eye movement measures based on fixation durations during first pass reading

243 indicated a strong preview benefit but no effect of color or highlighting. This was shown by the

244 reliably lower first fixation durations and gaze durations for un-degraded parafoveal presentation

245 in contrast to degraded previews (see Figure 1b). No reliable effects and interactions of color or

246 underlining were found (see Table 1). This finding indicates that bottom-up perceptual

247 processing preceding word recognition (i.e., in parafoveal vision) was only influenced by

248 degraded parafoveal previews but not reliably by word color or underlining.

249 The total viewing times, including all re-fixation times after the first encounter (i.e., re-

250 reading) and the go-past times (including all fixations up to the moment the next word in the

251 sentence is fixated), showed, in addition to a reliable degradation effect, a reliable interaction of

252 word color and underlining and a reliable effect of underlining, respectively. Figure $1 \mathrm{~b}$ clearly

253 shows the origin of the interaction in total viewing times and the underlining effect in go-past

254 times. The latter was indicated by lower go-past times for not underlined words in contrast to 
255 underlined words. The interaction for the total viewing times was reflected by the reduced

256 fixation times for un-highlighted black-presented words in contrast to all other conditions

257 including blue underlined, blue not-underlined and black underlined words (confirmed by post-

258 hoc analysis: underlining effect for black targets; estimate $=0.046 ; \mathrm{SE}=0.020 ; t=2.29$; no

259 underlining effect for blue targets; estimate $=0.002 ; \mathrm{SE}=0.016 ; t=0.12$ ). Both the interaction

260 in total viewing times and the underlining effect in go-past times indicate that highlighting

261 influences processing after first pass reading. The comparison of the two measures showed that

262 re-readings after the target word was passed (reflected by total viewing times) reduced the effect

263 of underlining for blue written words. This indicates that highlighting either by color or

264 underlining increases the total reading times reflecting the allocation of additional attentional

265 resources to highlighted words after the target word was first passed. Therefore, the reduced

266 skipping probability of blue target words, described by Fitzsimmons and colleagues (2013) in

267 their experiment one, might also reflect a highlighting effect for sentences in which target word

268 skipping can be realized to a higher extent. Finally, the finding of their second experiment in

269 which they observed longer go-past on blue low frequent words in contrast to black low frequent

270 words could be replicated (color effect for not underlined words irrespective of preview; go-past

271 times: estimate $=0.029 ; \mathrm{SE}=0.013 ; t=2.27$.

272 In sum, the present study demonstrated that reading was not hampered by blue text

273 presentation. Thus, the current findings do not indicate a bottom-up perceptual disadvantage of

274 blue underlined hypertext in foveal and parafoveal processing. In contrast, the increased total

275 viewing time for highlighted stimuli indicates an additional allocation of attentional resources

276 triggered by top-down processes. These processes might reflect the learned association of

277 hypertext to informative snips of texts in the Internet. Using blue underlined stimuli effectively 
278 highlights hypertext without hindering parafoveal and foveal perceptual processes during reading.

279 In addition, the present study shows that text underlining and color results in highlighting that

280 indicates that either could be used independently to optimally implement hypertext. In

281 conclusion, blue underlined hypertext allows effective reading and, therefore, can be safely

282 recommended to web designers for future use. 
285 I want to thank Arturo Hernandez for helpful discussions and comments on an earlier version of 286 the manuscript. I also want to thank Susanne Eisenhauer, Kirsten Hilger and Edvard Heikel for 287 comments on an earlier version and Agnes Altmanninger, Pia Schweitzer and Eva Daspelgruber 288 for helping with the data acquisition. Finally, I want to thank Florian Hutzler for letting me use 289 his laboratory and Stefan Hawelka since part of the current software used in the project was 290 scripted in collaboration for previous studies.

291

292

293

294

295

296

297

298

299

300

301 302

303 304 305

\section{References}

Auer, M., Gruber, G., Mayringer, H., \& Wimmer, H. (2004). Salzburger Lesescreening 5-8. Handbook. Salzburg: Mimeo.

Bates, D., Maechler, M., Bolker, B., Walker, S., Christensen, R. H. B., Singmann, H., ... \& Rcpp, L. (2015). Package 'lme4'. https://github.com/lme4/lme4/ http://lme4.r-forge.r-project.org/.

Brysbaert, M., Buchmeier, M., Conrad, M., Jacobs, A. M., Bölte, J., \& Böhl, A. (2011). The word frequency effect: A review of recent developments and implications for the choice of frequency estimates in German. Experimental Psychology, 58, 412-424.

Fitzsimmons, G., \& Drieghe, D. (2013). How fast can predictability influence word skipping during reading? Journal of Experimental Psychology: Learning, Memory, and Cognition, 39(4), 1054. 
307 Fitzsimmons, G., Weal, M., \& Drieghe, D. (2013). On measuring the impact of hyperlinks on reading. In Proceedings of the 5th Annual ACM Web Science Conference (pp. 65-74). ACM.

309

310 Gagl, B., Hawelka, S., \& Hutzler, F. (2014). A similar correction mechanism in slow and fluent 311 readers after suboptimal landing positions. Frontiers in human neuroscience, 8.

313 Gagl, B., Hawelka, S., Richlan, F., Schuster, S., \& Hutzler, F. (2014). Parafoveal preprocessing 314 in reading revisited: Evidence from a novel preview manipulation. Journal of Experimental 315 Psychology: Learning, Memory, and Cognition, 40(2), 588.

316

317 Hawelka, S., Schuster, S., Gagl, B., \& Hutzler, F. (2015). On forward inferences of fast and slow 318 readers. An eye movement study. Scientific reports, 5.

320 Hutzler, F., Fuchs, I., Gagl, B., Schuster, S., Richlan, F., Braun, M., \& Hawelka, S. (2013).

321 Parafoveal X-masks interfere with foveal word recognition: Evidence from fixation-related brain 322 potentials. Frontiers in System Neuroscience, 7, 33.

323

324 Kliegl, R., Hohenstein, S., Yan, M., \& McDonald, S. A. (2013). How preview space/time 325 translates into preview cost/benefit for fixation du- rations during reading. Quarterly Journal of 326 Experimental Psychology, 66, 581-600. 
328 Kliegl, R., Grabner, E., Rolfs, M., \& Engbert, R. (2004). Length, frequency, and predictability

329 effects of words on eye movements in reading. European Journal of Cognitive Psychology, 16(1330 2), 262-284.

331

332 Kliegl, R., Risse, S., \& Laubrock, J. (2007). Preview benefit and parafoveal-on-foveal effects

333 from word n+2. Journal of Experimental Psychology: Human Perception and Performance, $33433(5), 1250$.

335

336 Nielsen, J. (1999) When bad design elements become the standard. Nielsen Norman Group, 337 http://www.nngroup.com/articles/when-bad-design-elements-become-the-standard/

Radach, R., Huestegge, L., \& Reilly, R. (2008). The role of global top-down factors in local eyemovement control in reading. Psychological research, 72(6), 675-688.

342 Rayner, K. (1975). Perceptual span and peripheral cues in reading. Cognitive Psychology, 7, 6534381.

Richlan, F., Gagl, B., Schuster, S., Hawelka, S., Humenberger, J., \& Hutzler, F. (2013). A new

346 high-speed visual stimulation method for gaze-contingent eye movement and brain activity 347 studies. Frontiers in systems neuroscience, 7, 33.

349 Schotter, E. R., Angele, B., \& Rayner, K. (2012). Parafoveal processing in reading. Attention, 350 Perception, \& Psychophysics, 74(1), 5-35. 
351

352 Yarkoni, T., Balota, D., \& Yap, M. (2008). Moving beyond Coltheart's N: A new measure of 353 orthographic similarity. Psychonomic Bulletin \& Review, 15(5), 971-979. 